\title{
IMPORTANCIA DE LAS ESTRATEGIAS DE PRÁCTICA INSTRUMENTAL EN LA EDUCACIÓN MUSICAL SUPERIOR
}

\author{
Silvia Tripiana
}

Conservatorio Superior de Música de Aragón

silvtm@gmail.com

\begin{abstract}
El estudio tuvo por objeto observar el papel que ejercen determinadas estrategias de práctica instrumental. Mediante metodología observacional (formato de campo), se analizaron sesiones de práctica de 30 participantes que aplicaron en su estudio estrategias propuestas por la docente y sesiones de práctica libre de otros 30 individuos a los que no se les sugirió ninguna estrategia. Tras 30 minutos, el porcentaje de mejora de la obra musical con respecto al número de fallos registrados en la interpretación previa, fue de un $44.6 \%$ en el caso de la práctica estratégica; mientras que tras la práctica libre se registró un empeoramiento de un 5.9\%.

Palabras clave: estrategias de práctica instrumental, estudio individual, aprendizaje, instrumento.

The object of the study was the observation of the role of instrumental practice strategies. Through observational methodology (field format), practice sessions were analyzed of 30 participants who utilized practice strategies proposed by the teacher and by another 30 participants who were left to practice freely with no strategies suggested. After 30 minutes, the percentage of students who improved their performance, with respect to the number of mistakes logged before beginning the practice was $44.6 \%$ for the group developing practice strategies, while the students left to practice freely showed a decline in accuracy of $5.9 \%$.
\end{abstract}

Keywords: instrumental practice strategies, individual study, learning, instrument.

\section{INTRODUCCIÓN}

Un instrumentista profesional ha de tener presente que deberá ser capaz de gestionar la intensa tensión física y emocional que puede experimentar sobre el escenario por lo menos durante algunas fases de su carrera. Ello implica dominar complejas habilidades estratégicas. Algunas de estas habilidades están inexorablemente vinculadas a la técnica instrumental, mientras que otras estrategias pueden ser enseñadas con relativa independencia (LEHRER, 1987). En definitiva, se entiende que las estrategias de práctica instrumental son:

[...] aquellos pensamientos y comportamientos que, de forma consciente e intencional, guían al intérprete durante la práctica deliberada de su instrumento e intervienen en el modo en el que selecciona, organiza, procesa, integra y ejecuta sus conocimientos y habilidades musicales; en su estado emocional; y/o en su motivación; con el propósito de adquirir, almacenar y posteriormente poder reproducir resultados instrumentales, a su juicio, positivos y en el menor tiempo de consecución posible. (TRIPIANA, 2012:66)

En principio cabe pensar que un estudiante de instrumento que ingresa en el Grado Superior de Música, tras una media de 10 años de estudios musicales, debería tener una práctica efectiva y, por lo tanto, un manejo adecuado de un buen número de estrategias de práctica de avalada eficacia. Sin embargo, la experiencia docente permite comprobar que no es así en numerosos casos; en muchas ocasiones el estudiante no conoce las estrategias o bien, pese a conocerlas, no forman parte de su práctica habitual. Por otra parte, existen cuestiones de la literatura científica relacionada que ponen en alerta.

Las estrategias de práctica instrumental:

- Son generalmente poco conocidas. Varios estudios han puesto de manifiesto que los profesionales poseen un conocimiento limitado de las mismas (HALlaM, 2001; PITTS, DAVIDSON \& MCPHERSON, 2002) (RENWICK \& MCPHERSON, 2002).

- A veces se conocen pero apenas se utilizan. Byo y Cassidy (2008) demostraron que los estudiantes generalmente saben lo que tienen que hacer durante el estudio individual de su instrumento pero no necesariamente lo efectúan en su práctica habitual. 
- Poca relevancia durante el periodo de formación. Durante 3 años consecutivos, Jørgensen (2004) solicitó información a los estudiantes de nuevo ingreso en el conservatorio. Aproximadamente el $40 \%$ respondió que su profesorado anterior había puesto muy poco o ningún énfasis en las estrategias de estudio instrumental.

- Su proceso de enseñanza-aprendizaje no es necesariamente fácil. En el estudio de Barry y McArthur (1994), la mayoría de los docentes afirmaron que siempre o casi siempre discutían la importancia de la práctica y sus estrategias específicas con los estudiantes. Sin embargo, lo que el estudiante percibe y recuerda puede no corresponder con lo que el docente considera que ha comunicado. De hecho, Kostka (2002) observó la discrepancia entre lo que los docentes creen enseñar sobre la práctica y lo que realmente aprenden los estudiantes sobre la misma.

- El docente posee un papel determinante en su manejo. Un estudio de Barry y Hallam (2002) acerca de las interacciones estudiante-docente en clase y la práctica posterior individual, reveló que los hábitos de práctica de los estudiantes fueron poco influenciados por los consejos docentes. En cambio, aquello que el docente hizo realmente durante las clases (p. ej., permitir que el estudiante probara una determinada estrategia) ejerció una influencia mucho más profunda sobre la práctica individual de sus estudiantes que los mensajes verbales.

- Las tácticas de motivación son fundamentales en su implementación. Para ello puede consultarse la revisión efectuada por la autora acerca de estrategias motivacionales que pueden ser aplicadas al aprendizaje instrumental (TRIPIANA, 2016).

- Determinadas estrategias se usan más en unos instrumentos que en otros. El aprendizaje de los diversos instrumentos revela diferencias en cuanto a las estrategias de práctica que los docentes proponen para el estudio individual de su alumnado (GALICIA, ESPINO, HERNÁNDEZ, MERCADO, OROZCO, RAMÍREZ \& RUIZ, 2003).

Por todos estos motivos, se decidió estimular la práctica estratégica a través de indicaciones previas al estudio individual o, simplemente, observar cómo llevaban a cabo su estudio en solitario. El objetivo principal era analizar la efectividad de un programa didáctico sobre estrategias de práctica instrumental en los estudios superiores de música con instrumentistas de piano.

La aplicación de un programa didáctico sobre estrategias definidas de práctica instrumental en instrumentistas de piano complementario de estudios superiores de música mejora la interpretación de obras musicales. La hipótesis se plantea en comparación con el supuesto de que dicho programa no fuera necesario al suponer que los instrumentistas que acceden a Grado Superior ya poseen una práctica lo suficientemente efectiva.

\section{MÉTODO}

Entre las diversas opciones metodológicas existentes, se consideró que la metodología observacional era la más apropiada por diferentes motivos. Se caracteriza por la no intervención del sujeto investigador en el fenómeno de estudio y por la no restricción de las respuestas de los individuos, a través de las tareas o los instrumentos de evaluación, ya que el objetivo principal era la conducta generada espontáneamente preservando el contexto natural.

Por consiguiente, gracias a este procedimiento científico se ha obtenido información objetiva extraída de los comportamientos, episodios, actividades y situaciones que interesaban, y, a lo largo de las diversas fases constitutivas, se ha organizado, depurado y analizado la información registrada. De modo que, el proceso se constituyó con: planteamiento de objetivos, decisión de diseño observacional, muestreo, instrumentos, registro y codificación, obtención de parámetros, control de la calidad del dato, análisis de datos e interpretación de los resultados obtenidos. 


\section{Participantes}

Los agentes implicados fueron 60 estudiantes del Conservatorio Superior de Música de Aragón de 17 especialidades instrumentales diferentes que durante 2010-2011 cursaban la asignatura de Piano Complementario. Se formaron dos grupos: 30 participantes que efectuaron sesiones de práctica instrumental estratégica y otros 30 que llevaron a cabo sesiones de práctica libre. La Tabla 1 hace referencia a los 30 participantes que aplicaron en su estudio diversas estrategias propuestas por la docente distribuidos por sexo y curso.

\begin{tabular}{|l|l|l|}
\hline Sesión de práctica pianística estratégica & Hombres & Mujeres \\
\hline Primer curso: Piano Complementario I & 12 & 4 \\
\hline Segundo curso: Piano Complementario II & 6 & 8 \\
\hline Total de participantes & 30 & \\
\hline
\end{tabular}

Tabla 1: Instrumentistas participantes en la sesión de práctica estratégica por sexo y curso

A su vez, la Tabla 2 hace referencia a la especialidad de los mismos:

\begin{tabular}{|c|c|c|}
\hline & Instrumento & Estudiantes \\
\hline \multirow[t]{4}{*}{ Cuerda-Arco } & Violín & 2 \\
\hline & Viola & 2 \\
\hline & Violonchelo & 2 \\
\hline & Contrabajo & 2 \\
\hline \multirow[t]{5}{*}{ Viento-Madera } & Clarinete & 1 \\
\hline & Fagot & 2 \\
\hline & Flauta travesera & 2 \\
\hline & Flautín & 2 \\
\hline & Oboe & 1 \\
\hline \multirow[t]{5}{*}{ Viento-Metal } & Bombardino & 1 \\
\hline & Trombón & 2 \\
\hline & Trompa & 2 \\
\hline & Trompeta & 2 \\
\hline & Tuba & 2 \\
\hline \multirow[t]{3}{*}{ Otras especialidades } & Percusión & 2 \\
\hline & Instrumentos de púa & 1 \\
\hline & Saxofón & 2 \\
\hline
\end{tabular}

Nota. En total participaron en la sesión de práctica con estrategias propuestas 30 estudiantes.

Tabla 2: Participantes en la sesión de práctica estratégica según especialidad 
Por otra parte, la Tabla 3 hace referencia a los participantes que efectuaron una sesión de práctica libre, es decir, 30 estudiantes a los que no se les sugirió ninguna estrategia que utilizar, ni previamente habían tenido contacto con la docente.

\begin{tabular}{|l|l|l|}
\hline Sesión de práctica pianística libre & Hombres & Mujeres \\
\hline Primer curso: Piano Complementario I & 12 & 6 \\
\hline Segundo curso: Piano Complementario II & 4 & 8 \\
\hline Total de participantes & 30 & \\
\hline
\end{tabular}

Tabla 3: Instrumentistas participantes en la sesión de práctica pianística libre por sexo y curso

Asimismo, la Tabla 4 hace referencia a la especialidad de los mismos:

\begin{tabular}{|c|c|c|}
\hline & Instrumento & Estudiantes \\
\hline \multirow[t]{4}{*}{ Cuerda-Arco } & Violín & 2 \\
\hline & Viola & 2 \\
\hline & Violonchelo & 2 \\
\hline & Contrabajo & 2 \\
\hline \multirow[t]{5}{*}{ Viento-Madera } & Clarinete & 1 \\
\hline & Fagot & 2 \\
\hline & Flauta travesera & 2 \\
\hline & Flautín & 2 \\
\hline & Oboe & 1 \\
\hline \multirow[t]{5}{*}{ Viento-Metal } & Bombardino & 1 \\
\hline & Trombón & 2 \\
\hline & Trompa & 2 \\
\hline & Trompeta & 2 \\
\hline & Tuba & 2 \\
\hline \multirow[t]{3}{*}{ Otras especialidades } & Percusión & 2 \\
\hline & Instrumentos de púa & 1 \\
\hline & Saxofón & 2 \\
\hline
\end{tabular}

Nota. En total participaron en la sesión de práctica pianística libre 30 estudiantes

Tabla 4: Participantes en la sesión de práctica pianística libre según especialidad

En todo momento se tuvo en cuenta la heterogeneidad del nivel de los estudiantes en la asignatura de Piano Complementario, un alumnado que no mantiene una relación real con el piano de 10 años, aunque sí musical. No obstante, para garantizar los criterios de representatividad e idoneidad de la muestra, se buscó el equilibrio entre sujetos con mayor y menor nivel instrumental. Para ello se utilizó el criterio de cinco jueces expertos con Título Superior de Piano y docentes del Departamento de Piano del Conservatorio Superior de Música de Aragón. De modo que, en las audiciones de estudiantes de primer y segundo curso de Piano Complementario que tuvieron lugar 
previamente, se les solicitó que clasificaran a los instrumentistas participantes de dichas audiciones en tres grupos (nivel inicial/medio/experto), según fuera su interpretación. Finalmente, tras su oportuno juicio, se seleccionaron aquellos estudiantes que fueron clasificados en el mismo grupo por unanimidad.

\section{Instrumentos}

Se presentan los instrumentos que han formado parte del diseño:

\section{Programa didáctico basado en estrategias de práctica instrumental}

Un largo proceso de documentación y reflexión previo fue necesario para elaborar y desarrollar el programa didáctico sobre estrategias de práctica instrumental teniendo presentes las aportaciones del ámbito de Ciencias de la Educación, la Psicología Cognitiva y, muy particularmente, de la Didáctica de la Música.

Mientras que los programas de intervención basados en la evidencia científica son comunes en el deporte y en otros ámbitos disciplinares, no ocurre así dentro de la música y las artes escénicas. En parte esto es debido, según Clark y Williamon (2009), a que todavía existe poca evidencia científica que indique la diversa gama de beneficios que los artistas pueden obtener de este tipo de programas, así como los medios más eficaces con los que mejorar la práctica y la educación musical.

Una vez identificadas las 21 estrategias de práctica, avaladas por grandes figuras de la docencia instrumental, testimonios de grandes intérpretes y recientes investigaciones, a continuación, se pasó a elaborar el programa didáctico. La intención era que estuviera constituido por un conjunto de intervenciones organizadas en un plan de actuación no intensivo que se desarrollara en el contexto habitual de los participantes (en este caso, instrumentistas) usuarios del programa, sin tener carácter intrusivo (ANGUERA, 2003). Asimismo, sus fundamentos didácticos se hacen eco de ideas extraídas de investigaciones educativas encuadradas en el marco de la Psicología Cognitiva y en un enfoque naturalista de las Ciencias de la Educación, concretamente en el paradigma mediacional configurado en la Didáctica. Se trataba de generar actividades atractivas, variadas y capaces de motivar al estudiante más reacio a aprender y para ello era preciso que respondieran, en la medida sea posible y según Lucena (2008), a los intereses de los estudiantes. De modo que ha resultado importante que en su planificación se hayan tenido en cuenta las características (madurativas y sociales) de los mismos.

El objetivo primordial era obtener una interpretación musical resultante, tras la práctica bajo las directrices de dicho programa, que fuera satisfactoria para el instrumentista y, en la medida de lo posible, libre de parámetros alterados de precisión, continuidad, ritmo, agógica, dinámica, articulación, discurso musical, ornamentación, equilibrio corporal, aspecto gestual y signos de control. Para ello se trabajaron 21 estrategias y, aunque todas ellas pueden usarse eficazmente en cualquier momento de la práctica, se clasificaron según la fase del estudio de una obra musical en la que poseen mayor relevancia. En primer lugar, se enumeran las estrategias halladas que fueron consideradas de mayor interés al comenzar el estudio de una nueva obra:

- La primera estrategia propone una práctica fragmentada dividiendo la complejidad y progresando de lo sencillo a lo complejo.

- La segunda estrategia trata de practicar con máxima concentración durante la ejecución instrumental. 
- La tercera estrategia hace referencia a la adecuación ergonómica, al funcionamiento cómodo y natural del cuerpo del instrumentista por medio del movimiento natural, la economía motriz con máximo rendimiento y mínimo esfuerzo, la influencia de la gravedad, integrando la respiración y buscando el apoyo para obtener seguridad.

- La cuarta estrategia sugiere el manejo de mensajes constructivos de auto-orientación que plantean objetivos durante el estudio, sirven de auto-guía por medio de mensajes de advertencia, de apoyo o de recuerdo y efectúan una retroalimentación informativa evaluando los resultados o la sesión de práctica.

- La quinta estrategia tiene presente la escucha autocrítica, de modo que el instrumentista ha de percibir cómo cada sonido resuena en el espacio exterior, efectuando una adecuada retroalimentación y siendo ayudado por los medios audiovisuales.

- La sexta estrategia propone una lectura precisa tocando con corrección desde la primera vez, aislando la dificultad, analizando su naturaleza y asumiendo un margen de aceptabilidad sin obsesionarse con la perfección.

- La séptima estrategia supone una práctica indirecta alterando el tempo de la interpretación, generando nuevos problemas por resolver y acrecentando la dificultad.

- La octava estrategia sugiere al instrumentista una adaptación a su límite técnico-interpretativo actual, es decir, no pretender más de lo que en el momento es capaz de hacer.

A continuación, se presentan las estrategias que poseen mayor relevancia en la fase intermedia del estudio de una obra instrumental:

- La novena estrategia tiene por objeto la práctica del detalle por medio de la vigilancia continua de la calidad del sonido producido nota a nota (pequeña escala), prestando la máxima atención a los pormenores que tienen lugar frase a frase (media escala) y practicando la obra o el fragmento completo esperando la crítica para mejorar la calidad en su conjunto (gran escala).

- La décima estrategia está dedicada a la práctica técnica y pretende buscar el equilibrio entre la práctica constructiva y la práctica interpretativa así como manejar la técnica al servicio de la música.

- La estrategia décimo primera presenta el análisis, comprensión e interiorización armónicoformal como ayuda al instrumentista.

- La estrategia décimo segunda propone el análisis, comprensión e interiorización de los elementos expresivos de la obra: carácter, agógica, silencios, dinámica, elementos melódicos, timbre, afinación y elementos rítmicos.

- La estrategia décimo tercera sugiere el apoyo al discurso musical por medio del gesto respiratorio anacrúsico previo, creando líneas musicales con un fraseo coherente y completo, acentuando correctamente y a través de la eficacia del canto.

- La estrategia décimo cuarta propone la visualización creativa, es decir, anteponer el pensamiento a la acción, pensar e imaginar antes de tocar. De esta estrategia, se constatan diversas propuestas; se puede efectuar la visualización creativa para adoptar un modelo corporal experimentado desde el interior (propiocepción), para crear una imagen cinestésica previa, para controlar mentalmente el movimiento (dirigir y supervisar) y para experimentar la receptividad sensorial interrelacionando un resultado positivo con sensaciones internas. Esta práctica mental también puede generar una imagen sonora previa, de modo que sea posible escuchar la obra en la mente lejos del instrumento. A su vez, los símbolos e imágenes evocadoras, pueden propiciar 
la creación de un programa extra-musical o la generación de una imagen anticipada del tempo que también pueden ayudar al intérprete. Del mismo modo, la visualización creativa puede ser de utilidad para lograr una imagen escénica previa, es decir un escenario mental.

- La décimo quinta estrategia hace referencia a aquellos pensamientos, comportamientos, pautas de conducta, etc., que fomentan la espontaneidad durante la práctica.

Por último, estas son las estrategias halladas que se consideraron de mayor interés en la fase final del estudio de una obra instrumental:

- La estrategia décimo sexta propone experimentar la transmisión de vibraciones musicales del instrumento al cuerpo del instrumentista.

- La estrategia décimo séptima tiene por objeto la transmisión del contenido musical, es decir, practicar deliberadamente la comunicación de ideas musicales.

- La estrategia décimo octava propone efectuar una práctica de riesgo comenzando firmemente y evitando detenciones durante el período de ejecución.

- La estrategia décimo novena recomienda que el foco de atención se sitúe en el presente de la ejecución por medio de una práctica consciente.

- La vigésima estrategia plantea el uso de mensajes constructivos de auto-motivación para afianzar la confianza del propio intérprete, transformando su miedo en valentía y/o llevando a cabo un diálogo positivo consigo mismo.

- La última estrategia de práctica instrumental sugiere un manejo positivo de la crítica externa.

\section{Instrumento de observación}

La extraordinaria diversidad de conductas del músico susceptibles de ser sistemáticamente observadas durante la práctica instrumental obligó a prescindir de instrumentos estándar y, por el contrario, invertir el tiempo preciso para su construcción ad hoc. El instrumento de observación que se utilizó en el presente estudio fue el formato de campo ya que resultaba mucho más flexible y se adaptaba mejor a la multidimensionalidad del problema de investigación.

Según la perspectiva desde la cual se abordó el estudio, interesaron elementos de orden diverso: de aprendizaje, de destrezas o, incluso, de control del propio cuerpo. Ha de quedar claro que únicamente se registraron aquellos sectores del comportamiento que se podían percibir y que permitieron objetivar (y posteriormente cuantificar) su estudio. Indudablemente su alcance se hallaba únicamente restringido por la perceptibilidad y, en consecuencia, por la posibilidad de captar la ocurrencia de conductas a través de los órganos sensoriales (visuales y auditivos) mediante la grabación y almacenamiento de la información.

Dos de los niveles clásicos de respuesta han sido fundamentales en el presente estudio. Siendo consciente de sus limitaciones, estos se basan en la clasificación de niveles de respuesta de Weick (1968) y corresponden al contenido de la conducta a observar:

- Conducta no verbal: expresiones motoras que pueden originarse en distintas partes del cuerpo, para lo cual se adoptó la modulación introducida por Anguera (2001) que consiste en contemplar, como modalidades de la conducta no verbal, las expresiones faciales, la conducta gestual y la conducta postural.

- Conducta verbal o lingüística: se refiere al contenido de los mensajes. 
A su vez, debido a las características especiales del estudio musical, se tuvieron presentes otro tipo de conductas de interés:

- Conductas de interpretación musical: los intérpretes producen realizaciones físicas de ideas musicales. Dentro de ellas se distinguen:

- Conductas de fidelidad al texto: realización de determinados parámetros de la obra musical objetivos y compartidos por todos los intérpretes. Durante la interpretación se requiere que el intérprete ejecute las notas, silencios, ritmos, dinámicas, signos de articulación... correctos de una idea musical, en el caso de que exista una referencia (escrita o conceptual) con respecto a la cual se pueda medir la fidelidad (conductas perceptibles por el oído y/o la vista).

- Indicadores de expresión musical: además de la fidelidad textual, es deseable que los intérpretes den vida a la música, es decir, que vayan más allá de lo que indica explícitamente la partitura. Es lo que en términos musicales significa que "ser expresivo". Estas conductas a pesar de ser perceptibles auditivamente no han sido incluidas en la observación por su elevado grado de subjetividad y por la falta de criterio unánime entre intérpretes de distintas escuelas. Entre estas conductas se incluirían, por ejemplo, la calidad sonora, la proyección musical, el carácter, la comunicación, la convicción musical o criterios estilísticos.

\section{Instrumentos de registro}

Se tuvo en consideración que "la multiplicidad de aspectos del comportamiento en situación natural no puede ser aprehendida más que efectuando la selección de lo que va a ser observado" (QUERA, 1993:244), es decir, tan solo aquello relevante para el problema de investigación. En este sentido, resultó sumamente importante efectuar un planteamiento adecuado del registro.

En los últimos años, el avance tecnológico ha permitido el desarrollo de múltiples aplicaciones informáticas para registrar toda conducta perceptible. Entre las diferentes opciones, se optó por el programa Thèmecoder (JONSSON, 2003) que permite el registro a partir de grabaciones digitalizadas y resulta especialmente útil para generar ficheros que puedan ser importados al programa Thème (MAGNuSSON, 2005), utilizado en el análisis de datos para la detección de patrones temporales.

\section{Instrumentos técnicos}

Para llevar a cabo la presente investigación ha sido preciso un equipo de medios audiovisuales con grabación de audio y vídeo, una cámara de vídeo digital y un equipo informático.

\section{Instrumentos de análisis de datos}

Una vez que los datos superaron el control de calidad, debían someterse, según Anguera, Magnusson y Jonsson (2007), a una adecuada técnica de análisis, teniendo presente que el análisis de datos se encuentra condicionado al diseño planteado en cada caso.

En este sentido, se utilizó, por su adecuación al diseño y especial relevancia, la detección de $T$ Patterns (MAGNUSSON, 2005), "análisis que es posible aplicar siempre que interese conocer en qué medida existe alguna regularidad temporal relativa a la ocurrencia (o co-ocurrencia) de códigos, y se disponga de datos organizados de forma adecuada" (ANGUERA et al., 2007:74).

La detección de T-patterns se trata de una técnica de análisis desarrollada por Magnusson (2005), que permitió representar el dendrograma correspondiente a acciones compuestas de códigos concurrentes (configuraciones) que ocurrían en el mismo orden, con distancias temporales entre sí 
en cuanto a las unidades de tiempo utilizadas, que permanecían relativamente invariables dentro del intervalo crítico fijado con anterioridad.

El T-Pattern emerge fruto de un proceso matemático que, en forma de algoritmo, está automatizado en dicho programa informático Thème, desarrollado por Magnusson (2005) desde hace más de un cuarto de siglo y que realiza intensivos análisis estructurales que facilitan la difícil tarea de descubrir, describir y eventualmente explicar la compleja estructura intra e interindividual de la corriente de conducta. En la presente investigación se utilizó dicho programa en la versión 6 , que salió a la luz en abril de 2013 y que dispone de una versión educativa libre para su uso en investigación.

El tipo de patrones que Thème detecta son independientes de la escala temporal, de modo que el programa es capaz de analizar cualquier dato en el tiempo de unidades diversas (micro-segundos, horas, días, meses, o años). A su vez, los datos no necesitan estar basados en un tiempo determinado; solamente necesitan estar concentrados alrededor de uno o más niveles de respuesta. En este caso, los criterios seguidos para la detección de T-Patterns fueron que como mínimo su frecuencia de aparición fuera de tres ocasiones y su intervalo de confianza del $95 \%$.

\section{Procedimiento}

Treinta participantes aplicaron estrategias propuestas previamente por la misma docente en su sesión de práctica, mientras que otros 30 participantes efectuaron sus correspondientes sesiones de práctica libre donde no se les sugirió ninguna estrategia que utilizar. Todos ellos comenzaron tocando la obra musical completa, después practicaron durante media hora individualmente, unos con estrategias propuestas y otros de forma libre y, para finalizar, los participantes tocaron de nuevo la obra completa. De modo que entre la primera interpretación y la segunda transcurrieron, únicamente, los 30 minutos de práctica. A su vez, a la hora de diseñar el estudio se tuvo presente:

- Perfil de estudiantes: todos cursaban la asignatura de Piano Complementario y no habían sido aleccionados en estrategias de práctica instrumental por la docente-investigadora antes del estudio.

- Instrumento, piano: dada la condición de pianista de la docente-investigadora, es evidente que resultó más cercana la práctica en el propio instrumento; por ello se compararon sesiones de práctica pianística.

- Obras musicales: las distintas obras a interpretar se limitaron a aproximadamente una página de extensión con el fin de mantener la demanda de práctica de los estudiantes en un nivel semejante. No obstante, la misma obra fue objeto de estudio de un estudiante en sesión de práctica estratégica y de otro en sesión de práctica libre.

- Fase de aprendizaje similar: las sesiones de práctica se llevaron a cabo cuando el instrumentista consideraba que la obra era apta para una audición en clase ante la docente. Eso implicaba que, según su juicio, podía interpretarla de principio a fin con corrección, continuidad y precisión. Es evidente que cada instrumentista entendía esta premisa de diferente modo, según su anhelo de perfección, sus conocimientos previos, su nivel técnico-musical actual..., sin embargo, esto no significó un problema ya que lo importante era que el tiempo de práctica, con estrategias o libre, diera sus frutos.

- Tiempo de práctica: las sesiones tenían media hora de duración. 
Hay que tener presente que la docente observadora estuvo presente, sin intervenir, tanto en la interpretación inicial y final de las obras como en la sesión de práctica y que estas se llevaron a cabo en el aula de Piano Complementario del conservatorio.

\section{Diseño observacional utilizado}

La observación requirió ajustarse a unos requisitos para poderse materializar:

- Decisión de carácter sustantivo: delimitación temática del comportamiento perceptible del individuo que interesaba analizar y al cual afectaron tres únicas restricciones.

○ Carácter perceptible. En este estudio, se han considerado conductas manifiestas de los participantes que implicaran un grado total de perceptibilidad y que, por tanto, ofrecieran una mayor garantía para su acotación.

- Vida cotidiana del entorno natural de los implicados. En este estudio, la docente asumió el papel de investigadora y observadora no presencial de una práctica del alumnado dentro de su contexto cotidiano de actuación, a través de las filmaciones. Hay que tener presente que los estudiantes practican habitualmente en el aula donde se efectuaron las grabaciones.

- Relación interactiva con el entorno. En este estudio, se entiende por entorno el conjunto compuesto por los lugares (aula de instrumento) definidos en el espacio en que ocurren las diferentes actividades de la docente y el instrumentista.

- Decisiones desde el punto de vista procedimental o metodológico: el perfil básico de la metodología observacional.

- Carácter idiográfico ya que se ha tratado a los participantes de manera individual, en caso contrario hubiera sido nomotético.

- Ausencia de instrumento estándar. El carácter espontáneo de las conductas que se estudiaron, así como el carácter habitual del contexto, imposibilitó la existencia previa de unos instrumentos encorsetados y estándar que obligarían a limitar el carácter natural de las conductas producidas. Por dicho motivo, se construyeron ad hoc en cada caso.

En definitiva, se trata de un diseño idiográfico, puntual y multidimensional al contemplarse simultáneamente diversos niveles de respuesta dentro de una observación no participante. Este diseño está situado en el cuadrante II de la Figura 1. 


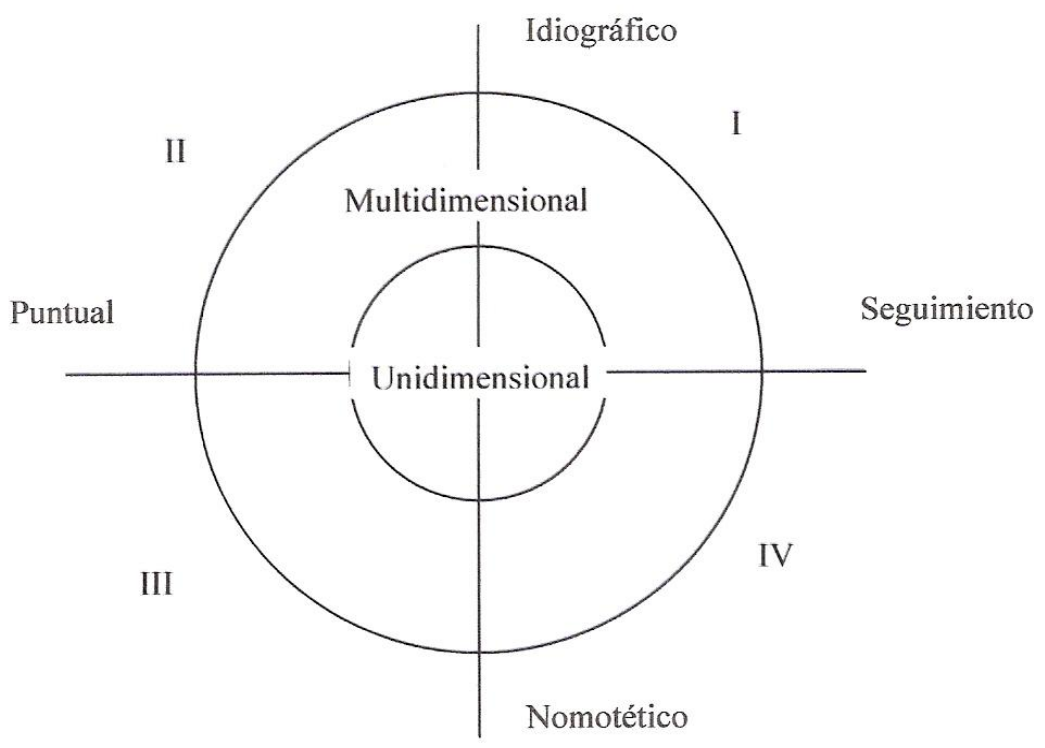

Figura 1. Diseños observacionales (Anguera, 2003).

\section{Reducción y eliminación de posibles sesgos}

En primer lugar, se tuvieron en consideración diversas medidas que trataron de reducir y eliminar los posibles sesgos de la metodología observacional (reactividad, sesgos de carácter técnico...). A su vez, para evitar sesgos de otra índole, se intentaron minimizar al máximo aquellos factores estresantes que podían afectar al instrumentista y se eliminó toda práctica instrumental fuera del período de observación.

\section{Elaboración de los instrumentos de observación}

En su elaboración se llevaron a cabo los siguientes pasos (ANGUERA, 2003):

- Establecimiento de criterios o ejes del instrumento, fijados en función del marco teórico y de los objetivos de la investigación. Estos criterios se clasificaron en cuatro apartados:

- Antes de comenzar a tocar la obra musical, se establecieron los criterios de:

- Postura corporal: posición de cabeza, tronco, espalda y extremidades del estudiante ante el instrumento.

- Estado corporal: situación de tensión o distensión en la que se encuentran los diversos grupos musculares del estudiante al colocarse ante el instrumento.

- Actitud gestual: forma de actuar del estudiante, con respecto a la seguridad y convicción en sus gestos, antes de comenzar a interpretar la obra musical. 
- Presencia escénica: este criterio hace referencia a la existencia o no de signos observables de miedo escénico en el estudiante antes de comenzar a interpretar la obra.

- Expresión verbal: mensajes verbales que emite el instrumentista antes de comenzar a tocar la obra musical.

- Anacrusa previa a la interpretación: este criterio hace referencia al gesto respiratorio anacrúsico que puede tener lugar antes de emitir el primer sonido de la obra.

- Durante la interpretación de la obra se establecieron diversos criterios en función de la correspondencia con el texto musical:

- Número de compás interpretado: este criterio hace referencia a la enumeración correlativa de compases de la partitura.

- Precisión: este criterio hace referencia a la exactitud en la ejecución de la notación con respecto a la partitura.

- Continuidad: este criterio hace referencia al desarrollo en el tiempo del texto musical que mantuvo el instrumentista.

- Ritmo: este criterio hace referencia a la exactitud en la ejecución de los valores rítmicos indicados en la partitura.

- Agógica: este criterio hace referencia a la exactitud en la ejecución de las indicaciones de tempo anotadas en la partitura.

- Dinámica: este criterio hace referencia a la exactitud en la ejecución de los matices de intensidad de sonido indicados en la partitura.

- Articulación: este criterio hace referencia a la exactitud en la ejecución de las indicaciones de articulación anotadas en la partitura.

- Discurso musical: este criterio hace referencia a la conducción del fraseo musical a través de la partitura.

- Ornamentación: este criterio hace referencia a la exactitud en la ejecución de las indicaciones de los ornamentos que aparecen en la partitura.

○ Durante la interpretación de la obra también se establecieron diversos criterios de la figura del intérprete:

- Equilibrio corporal: situación de tensión o distensión en la que se encuentran los diversos grupos musculares del estudiante mientras ejecuta la obra musical.

- Aspecto gestual: forma de actuar del estudiante conforme a la adecuación de sus gestos a la interpretación de la obra musical.

- Signos de control: forma de actuar del estudiante, con respecto a la seguridad y convicción en sus gestos, mientras interpreta la obra musical.

- Miedo escénico: este criterio hace referencia a la existencia de signos fisiológicos observables de Trac escénico en el estudiante durante la interpretación de la obra musical. 
- Expresión verbal: mensajes verbales que emite el instrumentista durante la ejecución de la obra musical.

- Después de la interpretación de la obra musical se establecieron los criterios de:

- Semblante: este criterio hace referencia a la existencia de signos observables de comunicación no verbal en el instrumentista, inmediatamente después de concluir la interpretación de la obra musical.

- Expresión verbal: mensajes verbales que emite el instrumentista inmediatamente después de concluir la interpretación de la obra musical.

- Catálogo de conductas/situaciones correspondientes a cada uno de los criterios. Esta lista fue señalada a partir de la información que proporcionó la fase exploratoria del estudio con, aproximadamente, 15 horas de sesiones de observación previas. Por otra parte, y al tratarse de una lista no cerrada, durante la visualización posterior de las grabaciones, se fueron añadiendo al catálogo aquellas nuevas conductas que iban apareciendo.

- Asignación de un sistema de codificación a cada una de las conductas/situaciones anotadas que se derivaron de cada uno de los criterios.

Tras este complejo proceso, se terminó de elaborar el instrumento de observación utilizado en el presente estudio y representado en la Figura 2.

Por último, para verificar la fiabilidad inicial del instrumento de observación construido, se llevó a cabo el control por medio de un segundo registro del mismo periodo al cabo de tres semanas y se compararon ambos registros para obtener evidencias de calidad en los datos (TÓJAR, 1993). Todos los datos se analizaron con el programa informático GSEQ 5.1. (QUERA, BAKEMAN \& GNISCI, 2007). Asimismo, resultó relevante registrar no solo la frecuencia de los eventos acaecidos sino también su orden de aparición y la duración de determinados parámetros, por ello se utilizó el coeficiente kappa de Cohen (1960) con resultados de acuerdo de un 99\% en el control de la calidad del dato del instrumento recientemente construido. 


\section{Antes de comenzar a tocar}

\begin{tabular}{|c|c|c|c|c|c|}
\hline Postura corporal & Estado corporal & Actitud gestual & Presencia escénica & Expresión verbal & Anacrusa previa \\
\hline Equilibrada & Relajación & Seguridad & Miedo escénico & Confianza & Con anacrusa \\
\hline Desequilibrada & Tensión & Inseguridad & $\ldots$ & Desconfianza & Sin anacrusa \\
\hline$\ldots$ & $\ldots$ & $\ldots$ & & $\ldots$ & $\ldots$ \\
\hline
\end{tabular}

\section{Durante la interpretación}

\begin{tabular}{|c|c|c|c|c|c|c|c|c|}
\hline Compás & Precisión & Continuidad & Ritmo & Agógica & Dinámica & Articulación & Discurso & Ornamento \\
\hline $\mathrm{C} 1$ & Error & Pausa & $\begin{array}{l}\text { Figura larga } \\
\text { mal medida }\end{array}$ & $\begin{array}{c}\text { Disminución del } \\
\text { tempo }\end{array}$ & Defecto dinámico & Defecto legato & $\begin{array}{c}\text { Acento } \\
\text { incorrecto }\end{array}$ & $\begin{array}{l}\text { No realiza } \\
\text { indicación }\end{array}$ \\
\hline $\mathrm{C} 2$ & $\begin{array}{l}\text { Defecto de } \\
\text { afinación }\end{array}$ & Ruptura de la línea & $\begin{array}{l}\text { Silencio largo } \\
\text { mal medido }\end{array}$ & Exceso de tempo & $\begin{array}{c}\text { Reguladores no } \\
\text { progresivos }\end{array}$ & Defecto staccato & Defecto fraseo & $\begin{array}{l}\text { Defecto de } \\
\text { ejecución }\end{array}$ \\
\hline $\mathrm{C} 3$ & $\ldots$ & Notas repetidas & Defecto rítmico & Rubato artificial & $\ldots$ & $\begin{array}{c}\text { Defecto } \\
\text { staccatissimo }\end{array}$ & $\begin{array}{c}\text { No avanza hacia } \\
\text { clímax }\end{array}$ & $\begin{array}{c}\text { Baja calidad del } \\
\text { ornamento }\end{array}$ \\
\hline $\mathrm{C} 4$ & & $\begin{array}{l}\text { Repetición de } \\
\text { fragmento }\end{array}$ & $\begin{array}{l}\text { Defecto } \\
\text { calderón }\end{array}$ & No realiza ritard. & & Defecto acento & $\cdots$ & $\ldots$ \\
\hline C5 & & $\ldots$ & $\ldots$ & No realiza accel. & & Defecto marcato & & \\
\hline C6 & & & & $\ldots$ & & Defecto Tenuto & & \\
\hline $\mathrm{C} 7$ & & & & & & Defecto portato & & \\
\hline$\cdots$ & & & & & & $\cdots$ & & \\
\hline
\end{tabular}


Durante la interpretación

\begin{tabular}{|c|c|c|c|c|c|}
\hline Compás & Equilibrio corporal & Aspecto gestual & Signos de control & $\begin{array}{c}\text { Signos de miedo } \\
\text { escénico }\end{array}$ & Expresión verbal \\
\hline C1 & Tensión facial & Gesto de negación & Comienzo firme & Sudoración profusa & Mensajes de auto-guía \\
\hline C2 & $\begin{array}{c}\text { Tensión en cintura } \\
\text { escapular }\end{array}$ & Gesto de apoyo métrico & Comienzo titubeante & Temblor facial & $\begin{array}{c}\text { Mensajes de auto- } \\
\text { motivación }\end{array}$ \\
\hline C3 & Tensión en codos & Gesto concordante & Recuperación óptima & Temblor manos & Duda \\
\hline C4 & Tensión en muñecas & Gesto no concordante & $\begin{array}{c}\text { Recuperación } \\
\text { deficiente }\end{array}$ & Frustración \\
\hline C6 & Tensión en dedos & $\begin{array}{c}\text { Respeto a los valores } \\
\text { largos }\end{array}$ & $\begin{array}{c}\text { Desplazamiento } \\
\text { controlado }\end{array}$ & & $\ldots$ \\
\hline$\ldots$ & $\ldots$ & $\begin{array}{c}\text { No respeto a los valores } \\
\text { largos }\end{array}$ & $\begin{array}{c}\text { Desplazamiento sin } \\
\text { control }\end{array}$ & & \\
\hline
\end{tabular}

\section{Después de la interpretación}

\begin{tabular}{|c|c|}
\hline Semblante & Expresión verbal \\
\hline Satisfacción & Mensaje satisfacción \\
\hline Insatisfacción & Mensaje insatisfacción \\
\hline Nerviosismo & Mensaje frustración \\
\hline Fatiga & Mensaje dubitativo \\
\hline Indiferencia & \\
\hline$\ldots$ & $\ldots$ \\
\hline
\end{tabular}

Figura 2. Instrumento de registro. 


\section{Registro y codificación}

La frecuencia ha sido tratada como un mero recuento de acciones u ocurrencias de conducta. Probablemente, haya sido el parámetro más utilizado tradicionalmente en observación y en este estudio ha quedado reflejado (p. ej., en el recuento de parámetros negativos observados en la interpretación) aunque, según Anguera (2008), es indudablemente el parámetro más débil.

El orden o explicitación de la secuencia de las diversas ocurrencias de conducta, ha resultado también de interés (p. ej., en el registro del orden de aparición de errores durante la interpretación). Además de contener la información correspondiente a la frecuencia ha permitido discriminar entre interpretaciones distintas que podrían aparecer como idénticas si solo se tuviera en consideración la frecuencia.

Finalmente, la duración resultó ser el parámetro de registro más consistente y el que encierra mayor riqueza de información, ya que contiene la del parámetro orden y además la indicación del número de unidades convencionales de tiempo (segundos) correspondientes a cada ocurrencia de conducta (p. ej., la duración de la interpretación de la obra).

\section{Control de la calidad del dato}

De las dos formas cuantitativas básicas para hallar la fiabilidad de los datos observacionales, en este estudio se utilizaron los coeficientes de acuerdo, resueltos mediante correlación.

A su vez, y para verificar la fiabilidad inicial del instrumento construido para observar la interpretación de las diversas obras musicales, se llevó a cabo un segundo registro del mismo periodo de observación al cabo de tres semanas. Todos estos datos se analizaron con el programa informático GSEQ 5.1. (QUERA et al., 2007).

En este instrumento era relevante registrar no solo la frecuencia de los eventos acaecidos sino también su orden de aparición y la duración de determinados parámetros con resultados del coeficiente kappa de $\kappa>.94$.

Posteriormente, durante la recogida de datos, se tuvo presente un exhaustivo control de la calidad del dato mediante el más básico de los requisitos de control o lo que tradicionalmente se ha denominado fiabilidad del registro observacional (ANGUERA, 2003).

\section{RESULTADOS}

Con este análisis se pretende justificar la necesidad de desarrollar programas didácticos sobre estrategias de práctica instrumental en los estudios superiores de música o si, por el contrario, no se considera necesario, puesto que el instrumentista que accede a Grado Superior ya posee una práctica lo suficientemente efectiva.

Los T-patterns obtenidos han permitido diferenciar diversos niveles de estructuras temporales complejas que se extienden a lo largo de los períodos de tiempo observados. De modo que, la consideración del orden, distancia temporal, posición y probabilidad media de ocurrencia de los eventos desvelaron las distintas estructuras ocultas subyacentes a la conducta interactiva de los participantes durante la práctica. Hay que tener presente que, la obtención del pattern ha resultado esencial para mostrar "la existencia de estructuras relacionales entre códigos, es decir, entre conductas que fueron registradas y codificadas, para ser después sometidas a un proceso de análisis cuantitativo" (ANGUERA et al., 2007:76). 
De modo que comparando los resultados obtenidos entre los estudiantes que efectuaron la práctica de modo libre y aquellos estudiantes que realizaron su práctica bajo las estrategias propuestas por la docente, se observa cómo estos últimos mejoraron substancialmente sus parámetros negativos. En estos estudiantes se registraron menos defectos (p. ej., notas falsas, ritmos imprecisos, tensiones ...) en la interpretación final de la obra musical con respecto a la interpretación inicial de la misma (Figura 3). Mientras que un estudiante que realizaba la práctica estratégica mejoraba 60.1 parámetros negativos de media; aquel que estudiaba de forma libre empeoraba de media 5.9 parámetros negativos. No obstante, como todos los estudiantes no partían de la misma situación, se calculó el porcentaje relativo. El porcentaje de mejora con respecto al número de fallos iniciales en el caso de la práctica estratégica fue de un 44.6\%; mientras que en el caso de la práctica libre se registró un empeoramiento de un $5.9 \%$.

PRÁCTICA LIBRE

\begin{tabular}{|c|c|c|c|c|c|c|c|c|}
\hline Obras & Inicio & Final & Mejora & \% Relativo & Inicio & Final & Mejora & \% Relativo \\
\hline Haydn: Danse Allemande & 50 & 67 & -17 & -34 & 200 & 92 & 108 & 54 \\
\hline Prokofieff: Marche & 97 & 110 & -13 & $-13,40206186$ & 106 & 48 & 58 & 54,71698113 \\
\hline Emonts: The little Cellist & 173 & 147 & 26 & 15,02890173 & 117 & 80 & 37 & 31,62393162 \\
\hline Grieg: op. $12 n^{o} 2$ & 166 & 197 & -31 & $-18,6746988$ & 320 & 239 & 81 & 25,3125 \\
\hline Satie: Gnossienne $n^{o} 1$ & 127 & 156 & -29 & $-22,83464567$ & 148 & 74 & 74 & 50 \\
\hline Granados: Oriental & 276 & 247 & 29 & 10,50724638 & 263 & 113 & 150 & 57,03422053 \\
\hline Rachmaninoff: Preludio & 183 & 154 & 29 & 15,84699454 & 97 & 55 & 42 & 43,29896907 \\
\hline Musorgsky: A tear & 141 & 201 & -60 & $-42,55319149$ & 115 & 64 & 51 & 44,34782609 \\
\hline Piazzolla: Invierno & 98 & 89 & 9 & 9,183673469 & 82 & 63 & 19 & 23,17073171 \\
\hline Bach: Preludio Do $M$ & 93 & 112 & -19 & $-20,43010753$ & 140 & 49 & 91 & 65 \\
\hline Tansman: Petite rêverie & 144 & 159 & -15 & $-10,41666667$ & 87 & 38 & 49 & 56,32183908 \\
\hline Handel: Sarabande & 154 & 153 & 1 & 0,649350649 & 176 & 97 & 79 & 44,88636364 \\
\hline Schubert: Menuetto & 120 & 169 & -49 & $-40,83333333$ & 236 & 126 & 110 & 46,61016949 \\
\hline Heller: Estudio $n^{\circ} 23$ & 116 & 128 & -12 & $-10,34482759$ & 111 & 62 & 49 & 44,14414414 \\
\hline Chopin: Preludio & 102 & 135 & -33 & $-32,35294118$ & 136 & 60 & 76 & 55,88235294 \\
\hline Granados: Asturias & 137 & 137 & 0 & 0 & 170 & 91 & 79 & 46,47058824 \\
\hline Tchaikovsky: Mazurka & 132 & 132 & 0 & 0 & 89 & 57 & 32 & 35,95505618 \\
\hline Mendelssohn: Romanza & 72 & 73 & -1 & $-1,388888889$ & 84 & 42 & 42 & 50 \\
\hline Haydn: Scherzo XVI/9 & 75 & 64 & 11 & 14,66666667 & 164 & 132 & 32 & 19,51219512 \\
\hline Albéniz: Rumores Caleta & 100 & 101 & -1 & -1 & 56 & 34 & 22 & 39,28571429 \\
\hline Satie: II Gnossienne & 133 & 150 & -17 & $-12,78195489$ & 62 & 35 & 27 & 43,5483871 \\
\hline Piazzolla: Milonga & 116 & 107 & 9 & 7,75862069 & 146 & 73 & 73 & 50 \\
\hline Mendelssohn: Gondellied & 100 & 133 & -33 & -33 & 108 & 61 & 47 & 43,51851852 \\
\hline Bach: Invención n ${ }^{\circ} 13$ & 183 & 178 & 5 & 2,732240437 & 84 & 44 & 40 & 47,61904762 \\
\hline Shoenmehl: Le joueur & 78 & 66 & 12 & 15,38461538 & 147 & 59 & 88 & 59,86394558 \\
\hline Lecuona: La comparsa & 113 & 128 & -15 & $-13,27433628$ & 101 & 42 & 59 & 58,41584158 \\
\hline Chopin: Mazurca op. 30 & 82 & 89 & -7 & $-8,536585366$ & 131 & 107 & 24 & 18,32061069 \\
\hline Bastien: Blues & 51 & 31 & 20 & 39,21568627 & 118 & 36 & 82 & 69,49152542 \\
\hline Granados: Vals Poético & 198 & 151 & 47 & 23,73737374 & 150 & 84 & 66 & 44 \\
\hline Albéniz: Malagueña & 152 & 177 & -25 & $-16,44736842$ & 107 & 91 & 16 & 14,95327103 \\
\hline PROMEDIO TOTAL & & & -5.966666667 & -5.9186746 & & & 60.1 & 44.57682436 \\
\hline
\end{tabular}

Figura 3. Parámetros negativos registrados en la observación de la interpretación. 
Por otra parte, se consideró interesante observar la interpretación de las obras antes y después del periodo de práctica instrumental desde dos perspectivas. En primer lugar, en la Figura 4, en cuanto a la correspondencia con la partitura (precisión, continuidad, ritmo, agógica, dinámica, articulación, discurso musical y ornamentación).

\begin{tabular}{|c|c|c|c|}
\hline \multicolumn{2}{|c|}{ PRÁCTICA LIBRE } & \multicolumn{2}{c|}{$\begin{array}{c}\text { PRÁCTICA CON ESTRATEGIAS } \\
\text { PROPUESTAS }\end{array}$} \\
\hline $\begin{array}{c}\text { PARÁMETROS } \\
\text { MEJORADOS }\end{array}$ & $\begin{array}{c}\text { PORCENTAJE DE } \\
\text { MEJORA } \\
\text { RELATIVO }\end{array}$ & $\begin{array}{c}\text { PARÁMETROS } \\
\text { MEJORADOS }\end{array}$ & $\begin{array}{c}\text { PORCENTAJE DE } \\
\text { MEJORA } \\
\text { RELATIVO }\end{array}$ \\
\hline-0.5 & $-6.1 \%$ & 26.7 & $50.3 \%$ \\
\hline
\end{tabular}

Figura 4. Correspondencia con la partitura.

En segundo lugar, la Figura 5 hace referencia a la observación de la figura del intérprete; equilibrio corporal, aspecto gestual, signos de control, miedo escénico y expresión verbal:

\begin{tabular}{|c|c|c|c|}
\hline \multicolumn{2}{|c|}{ PRÁCTICA LIBRE } & \multicolumn{2}{c|}{$\begin{array}{c}\text { PRÁCTICA CON ESTRATEGIAS } \\
\text { PROPUESTAS }\end{array}$} \\
\hline $\begin{array}{c}\text { PARÁMETROS } \\
\text { MEJORADOS }\end{array}$ & $\begin{array}{c}\text { PORCENTAJE DE } \\
\text { MEJORA } \\
\text { RELATIVO }\end{array}$ & $\begin{array}{c}\text { PARÁMETROS } \\
\text { MEJORADOS }\end{array}$ & $\begin{array}{c}\text { PORCENTAJE DE } \\
\text { MEJORA } \\
\text { RELATIVO }\end{array}$ \\
\hline-5.5 & $-8.3 \%$ & 33.4 & $40.9 \%$ \\
\hline
\end{tabular}

Figura 5. Observación de la figura del intérprete.

En cuanto a los estudiantes que practicaron de forma libre, se observa que no solucionaron ni empeoraron ningún parámetro correspondiente con la fidelidad a la partitura. En cambio, si se vieron afectados, relativamente más $(8.3 \%)$, en aquellos parámetros que atañen a la observación del intérprete. Si se observan los fallos de fidelidad al texto (precisión, continuidad, ritmo, agógica, dinámica, articulación, discurso musical y ornamentación) entre la obra interpretada antes y después de la sesión de práctica, se observa cómo los estudiantes que realizaron la práctica con estrategias mejoraron en un porcentaje relativo mucho mayor (50.3\%).

Para comprobar la incidencia de la dificultad de las obras en la práctica instrumental, se dividieron en tres grupos en función de su mayor o menor complejidad según el criterio de cinco jueces. Los resultados pueden observarse en la Figura 6: 


\begin{tabular}{|c|c|c|c|c|}
\cline { 2 - 5 } \multicolumn{1}{c|}{} & \multicolumn{2}{c|}{ PRÁCTICA LIBRE } & \multicolumn{2}{c|}{$\begin{array}{c}\text { PRÁCTCA CON ESTRATEGIAS } \\
\text { PROPUESTAS }\end{array}$} \\
\hline $\begin{array}{c}\text { DIFICULTAD } \\
\text { OBRA }\end{array}$ & $\begin{array}{c}\text { PARÁMETROS } \\
\text { MEJORADOS }\end{array}$ & $\begin{array}{c}\text { PORCENTAJE } \\
\text { DE MEJORA } \\
\text { RELATIVO }\end{array}$ & $\begin{array}{c}\text { PARÁMETROS } \\
\text { MEJORADOS }\end{array}$ & $\begin{array}{c}\text { PORCENTAJE } \\
\text { DE MEJORA } \\
\text { RELATIVO }\end{array}$ \\
\hline BAJA & -0.7 & $-0.4 \%$ & 62.6 & $49.0 \%$ \\
\hline MEDIA & -11.8 & $-11.2 \%$ & 49.1 & $41.4 \%$ \\
\hline ALTA & -5.4 & $-6.1 \%$ & 68.6 & $43.2 \%$ \\
\hline
\end{tabular}

Figura 6. Dificultad de la obra interpretada.

Los estudiantes que practicaban obras de dificultad baja de forma libre, no empeoraron pero tampoco mejoraron su resultado tras su estudio ( $-0.4 \%$ frente al $-5.9 \%$ general). Sin embargo, los estudiantes que practicaron de forma estratégica obras de dificultad baja mejoraron su rendimiento en un porcentaje relativo superior al registrado de forma general (49\% frente al $44.6 \%$ general). Por otra parte, durante la práctica libre las obras de dificultad media empeoraron mucho más $(-11.2 \%$ frente al $-5.9 \%$ general) y los estudiantes que realizaron la práctica estratégica mejoraron relativamente menos (41.4\% frente al $44.6 \%$ general).

Para comprobar si existían diferencias según el grado de corrección al interpretar la obra inicial, se dividieron los participantes en otros tres grupos. Los que en la obra inicial habían cometido menos de 100 errores; los que habían cometido entre 100 y 149, y por último, los que habían cometido 150 o más parámetros negativos en la interpretación de la obra inicial.

\begin{tabular}{|c|c|c|c|c|}
\cline { 2 - 5 } \multicolumn{1}{c|}{} & \multicolumn{2}{c|}{ PRÁCTICA LIBRE } & \multicolumn{2}{c|}{$\begin{array}{c}\text { PRÁCTICA CON ESTRATEGIAS } \\
\text { PROPUESTAS }\end{array}$} \\
\hline $\begin{array}{c}\text { INTERPRETACIÓN } \\
\text { INICIAL } \\
\text { NEGATIVOS }\end{array}$ & $\begin{array}{c}\text { PARÁMETROS } \\
\text { MEJORADOS }\end{array}$ & $\begin{array}{c}\text { PORCENTAJE } \\
\text { DE MEJORA } \\
\text { RELATIVO }\end{array}$ & $\begin{array}{c}\text { PARÁMETROS } \\
\text { MEJORADOS }\end{array}$ & $\begin{array}{c}\text { PORCENTAJE } \\
\text { DE MEJORA } \\
\text { RELATIVO }\end{array}$ \\
\hline $\begin{array}{c}\text { BAJOS } \\
\text { (inferior a 100 fallos) }\end{array}$ & -0.5 & $-0.08 \%$ & 34.1 & $42.4 \%$ \\
\hline $\begin{array}{c}\text { MEDIOS } \\
\text { (de 100 a 149 fallos) }\end{array}$ & -19.6 & $-16.3 \%$ & 58.9 & $47.2 \%$ \\
\hline $\begin{array}{c}\text { ALTOS } \\
\text { (150 o más fallos) }\end{array}$ & 10.1 & $4.2 \%$ & 88.1 & $42.2 \%$ \\
\hline
\end{tabular}

Figura 7. Parámetros negativos en la obra de inicio. 
Según la Figura 7, la práctica con estrategias fue más efectiva (con un mayor porcentaje relativo de mejora de parámetros previamente alterados) en aquellos instrumentistas que en la obra de inicio habían cometido entre 100 y 149 errores (47.2\% frente al 44.6\% general). En cambio, la sesión de practica libre fue más efectiva en aquellos que en la obra de inicio habían cometido entre 150 o más fallos (4.2\% frente al $-5.9 \%$ general) y empeoró (con un menor porcentaje relativo de mejora de parámetros previamente alterados) en aquellos que de inicio habían cometido entre 100 y 149 fallos $(-16.3 \%)$.

\section{DISCUSIÓN}

A la hora de discutir los resultados obtenidos entre los estudiantes que efectuaron la práctica de modo libre y aquellos estudiantes que aplicaron en su estudio diversas estrategias propuestas por la docente, hay que tener en cuenta que, en ambos casos, los estudiantes no habían recibido información acerca de las estrategias de práctica instrumental por parte de la docente-investigadora, aunque no se pudo descartar su formación previa sobre las mismas.

\section{Discusión de resultados}

Al analizar los resultados obtenidos, entre los estudiantes que efectuaron la práctica de modo libre y aquellos que realizaron su práctica con estrategias, se observa cómo estos últimos, al interpretar la obra tras la sesión de estudio, redujeron substancialmente los defectos cometidos en la interpretación inicial de la misma, mientras que los que estudiaban de forma libre no solo no mejoraban sino que empeoraban su resultado interpretativo final. Estos hallazgos se corresponden con los obtenidos en el estudio de 61 instrumentistas efectuado por Barry (1990) en el que aquellos estudiantes que utilizaron un enfoque estructurado para la práctica (con estrategias propuestas por el docente o de diseño propio) fueron capaces de corregir más errores interpretativos que los estudiantes que no seguían una práctica estructurada.

En la actual investigación, los estudiantes que practicaron libremente basaron su ensayo principalmente en la repetición, al igual que en los diversos estudios revisados por Parkes (2010). Sin embargo, a la vista de los resultados y coincidiendo con los obtenidos por Pitts et al. (2002) y con Parkes (2010), la repetición en sí misma no resultó efectiva si no se asoció a un objetivo y una determinada estrategia o serie de estrategias para incrementar significativamente el nivel de resultados. No obstante, cabe señalar que el mal resultado de los estudiantes que practicaron de forma libre pudo ser debido a la falta de confianza en la calidad y efectividad de la sesión de práctica mantenida. Este hecho ya quedó reflejado en el estudio con 53 pianistas de Kendrick, Craig, Lawson y Davidson (1982) que demostraron la importancia de la práctica con estrategias con objeto de incrementar la confianza del intérprete. En la presente investigación, los instrumentistas que practicaron libremente evidenciaron una falta de confianza en la calidad de su estudio que se tradujo en un exceso de tensión y nerviosismo durante la ejecución de la obra final. Mientras tanto, los estudiantes que siguieron las estrategias propuestas por la docente, al estar más convencidos de la calidad de su periodo de estudio, no presentaron este fenómeno por lo que la mejora resultó ser más significativa.

Del mismo modo, resultó interesante analizar la interpretación de las obras musicales desde dos perspectivas: observando la figura del instrumentista durante la ejecución y observando la correspondencia de lo interpretado con la partitura. 
En cuanto a los estudiantes que practicaron de forma libre, se evidencia que empeoraron relativamente más en aquellos parámetros que atañen a la observación del intérprete durante la ejecución (p. ej., incomodidad gestual, tensión muscular...). Esto implica que se mostraron mucho más ansiosos de demostrar que su práctica libre había sido efectiva y, como consecuencia, presentaron mayor tensión física en la interpretación de la obra final.

En cambio, si se analiza la correspondencia de lo interpretado con la partitura (p.ej., precisión en las notas, ritmo correcto...) entre la obra ejecutada antes de comenzar la práctica y la obra tocada al final de la misma, se observa cómo los estudiantes que realizaron la práctica estratégica mejoraron en un porcentaje relativo mucho mayor. De modo que se puede afirmar que las estrategias de práctica instrumental son más efectivas para subsanar los errores de fidelidad al texto que aquellos que afectan directamente al estado físico del intérprete. Un resultado perfectamente comprensible ya que, generalmente, según mi experiencia como docente y como pianista, se requiere mucho tiempo para subsanar este tipo de defectos que conciernen a la naturaleza corporal del músico.

No obstante, si se analizan los datos en función de la dificultad de las obras interpretadas, se observan otras cuestiones de interés. Los estudiantes que practicaron de forma estratégica obras de dificultad baja mejoraron su rendimiento en un porcentaje relativo superior al registrado de forma general. Por otra parte, los estudiantes que realizaron práctica estratégica con obras de dificultad media mejoraron relativamente menos. Por ello se puede concluir que el uso de estrategias durante la práctica es más efectivo en las obras de baja dificultad y es menos eficaz en las obras de dificultad media. Este hecho también resulta comprensible, ya que si un estudiante toca una obra de baja dificultad es porque posee un nivel pianístico escaso y es conocido que el uso de estrategias de práctica instrumental se incrementa con la experiencia, según Sloboda, Davidson, Howe y Moore (1996).

A su vez, se comprueba que existen diferencias según el grado de corrección al interpretar la obra antes de la sesión de estudio. La práctica estratégica fue más efectiva en aquellos estudiantes que tenían un nivel medio de parámetros negativos de inicio. En cambio, la práctica libre fue más efectiva en aquéllos que en la obra de inicio habían cometido más fallos y empeoró en aquéllos otros que de inicio habían cometido un nivel medio de fallos. Esto explica que, al comenzar a estudiar una obra, cualquier tipo de estudio, sea estratégico o no, obtiene resultados con prontitud; sin embargo, a la hora de mejorar una obra situada en su fase intermedia de estudio, la práctica con estrategias confiere mayores resultados de calidad que una práctica libre basada en la mera repetición de fragmentos sin un objetivo definido.

\section{Limitaciones del estudio y recomendaciones para posteriores investigaciones}

Llegados a este punto es preciso hacer mención a las limitaciones del estudio. En primer lugar hay que tener presente que la intervención del programa tuvo una duración muy breve. Sin embargo, este factor puede hacer doblemente prometedores sus resultados si se llevara a cabo con más tiempo. Asimismo, la muestra fue limitada, uno o dos estudiantes por especialidad instrumental. Con un mayor número de observadores se hubiera podido mantener una muestra más amplia de cada instrumento y conocer con mayor certeza el impacto del programa didáctico en cada una de las especialidades. Sin embargo, se optó por obtener una representación que fuera, razonablemente, asumible para una única observadora.

No obstante, el tema presenta distintas líneas argumentales y cada una de ellas abre nuevas y variadas vías para estudios posteriores. La primera vía de interés radica en el proceso de incorporación de las diversas estrategias de práctica en función del desarrollo evolutivo del 
estudiante de instrumento ya que, probablemente, sus posibilidades de manejo difieran en función de si el usuario de las mismas se trata de un niño, un joven o de un adulto.

La segunda vía implicaría comprender cómo el estudiante, incorpora nuevas estrategias a su práctica habitual y cómo optimiza sus ensayos y mejora su rendimiento durante la actuación pública.

Otra de las vías de interés se fundamentaría en la observación de sesiones de práctica de grandes intérpretes, para comprender con detalle cómo manejan las diversas estrategias, si pueden identificarse otras nuevas, cuáles son las más requeridas y cómo evolucionan en función de la fase de estudio en la que se encuentra la obra musical.

\section{Conclusiones}

En definitiva, y a la vista de los resultados, la investigación coincide con aquellos estudios que manifiestan que los instrumentistas profesionales poseen un conocimiento limitado de estrategias específicas de práctica instrumental (HALlam, 2001; PitTs et al, 2002; RENWICK \& MCPHERSON, 2002) o con aquellos otros trabajos que demostraron que los estudiantes generalmente conocen lo que tienen que hacer durante el estudio individual de su instrumento pero no necesariamente lo efectúan en su práctica habitual (BYO \& CASSIDY, 2008). Sin embargo, al igual que sostiene el estudio de Marín, Pérez y Hallam (2009), en todos los niveles existen una serie de diferencias individuales en la medida en que las estrategias son adecuadas, aplicables y eficaces.

De modo que, gracias a este análisis comparativo entre la práctica con estrategias propuestas y la práctica libre, se observa que en la mayor parte de los casos no es suficiente la formación previa recibida, lo que justifica la necesidad de implementar un programa didáctico sobre estrategias de práctica instrumental en la formación superior del estudiante. En definitiva, se confirma la necesaria implicación del docente de asignaturas instrumentales en la práctica del estudiante.

Para finalizar, a modo de conclusión:

- Los estudiantes que practican con estrategias de práctica instrumental, mejoran substancialmente su interpretación e incrementan su motivación por el logro de resultados musicales de calidad, evidenciando su satisfacción. Sin embargo, en todos los niveles existen una serie de diferencias individuales en la medida en que las estrategias son adecuadas, aplicables y eficaces.

- No obstante, las estrategias de práctica instrumental son más eficaces subsanando errores de fidelidad al texto; su uso es más efectivo en obras de baja dificultad, ya que el manejo de las mismas se incrementa con la experiencia; y obtienen mayores resultados a la hora de mejorar una obra situada en su fase intermedia de estudio, frente a la práctica libre basada en la mera repetición de fragmentos sin objetivo definido.

- El instrumentista, tras poner en práctica determinadas estrategias, ha logrado una interpretación final de la obra musical y, en ocasiones, la ha dado por válida a pesar de la existencia de parámetros alterados. Estas secuencias de errores o patrones temporales de conducta del estudiante, que no pueden ser explicados por el azar, han resultado ser muy relevantes para corroborar la eficacia de las estrategias ante diversos parámetros que intervienen en una interpretación musical (precisión, continuidad, ritmo, agógica, dinámica, articulación, discurso musical, ornamentación, equilibrio corporal, aspecto gestual y signos de control). 
- Las estrategias de práctica instrumental se posicionan en un plano fundamentalmente interdisciplinar e incluso transdisciplinar, en la medida en que ponen en juego la planificación y regulación consciente de acciones, pensamientos, pautas de conducta... dirigidas a un objetivo musical, superando el plano de lo meramente académico e incidiendo directamente en la práctica profesional.

En cuanto a la hipótesis inicial, se corrobora que los instrumentistas que acceden a Grado Superior poseen un bagaje de estrategias de práctica instrumental insuficiente, así como un conocimiento limitado de las mismas, lo que justifica la necesidad de implementar un programa didáctico sobre dichas estrategias.

Finalmente, la investigación concluye que el docente debe generar oportunidades de aplicación de práctica efectiva, deliberada e intensa en el aula adecuadas a cada individuo en función de su nivel de destreza. En definitiva, se confirma que las estrategias de práctica instrumental deben formar parte de las competencias transversales de la formación superior del instrumentista profesional.

\section{REFERENCIAS BIBLIOGRÁFICAS}

Anguera, M. T. (2001). Cómo apresar las competencias del bebé mediante una aplicación de la metodología observacional. Contextos educativos, 4, 13-64.

Anguera, M. T. (2003). La observación. En C. Moreno (Ed.), Evaluación psicológica. Concepto, proceso y aplicación en las áreas del desarrollo y de la inteligencia. Madrid: Sanz y Torres.

Anguera, M. T. (2008). Diseños evaluativos de baja intervención. En M. T. Anguera, S. Chacón y A. Blanco (Coords.), Evaluación de Programas Sociales y Sanitarios. Madrid: Síntesis.

Anguera, M. T., Magnusson, M. S., y Jonsson, G. K. (2007). Instrumentos no estandar: planteamiento, desarrollo y posibilidades. Avances en medición, 5, 63-82.

Barry, N. H. (1990). The effects of different practice techniques upon technical accuracy and musicality in student instrumental music performance. Research Perspectives in Music Education, $1,4-8$.

Barry, N.H., \& Hallam, S. (2002). Practice. En R. Parncutt \& G.E. McPherson (Eds.), The Science and Psychology of Music Performance: Creative Strategies for Teaching and Learning. Oxford: Oxford University Press.

Barry, N.H., \& McArthur, V. (1994). Teaching practice strategies in the music studio: A survey of applied music teachers. Psychology of Music, 22, 44-55.

Byo, J., \& Cassidy, J. W. (2008). An exploratory study of time use in the practice of music majors self-report and observation analysis. Update: Applications of Research in Music Education, 27(1), 33-40.

Clark, T. \& Williamon, A. (2009). Developing evidence-based interventions to enhance performance. En Williamon, A., Pretty, S. \& Buck, R. (Eds.), Proceedings of the International Symposium on Performance Science 2009. Utrecht, The Netherlands: European Association of Conservatoires (AEC).

Cohen, J. A. (1960). Coefficient of agreement for nominal scales. Educational and Psychological Measurement, 20, 37-46. 
Galicia, I. X., Espino, V., Hernández, E., Mercado, M., Orozco, R., Ramírez, A. y Ruiz, A. (2003). Estrategias de estudio promovidas por profesores de piano e instrumentos de aliento y cuerdas. Cuadernos Interamericanos de Investigación en Educación Musical, 2(5), 97-106.

Hallam, S. (2001). The development of expertise in young musicians: Strategy use, knowledge acquisition and individual diversity. Music Education Research, 3(1), 7-23.

Jonsson, G. K. (2003). ThèmeCoder, v4.0, [programa informático]. Noldus: PatternVision.

Jørgensen, H. (2004). Strategies for individual practice. En A. Williamon (Ed.), Musical excellence. Strategies and techniques to enhance performance. London: Oxford University Press.

Kendrick, M. J., Craig, K. D., Lawson, D. M., \& Davidson, P. O. (1982). Cognitive and behavioral therapy for musical-performance anxiety. Journal of Consulting and Clinical Psychology, 50, 353-362.

Kostka, M. J. (2002). Practice expectations and attitudes: a survey of college-level music teachers and students. Journal of Research in Music Education, 50(2), 145-154.

Lehrer, P. M. (1987). A Review of the Approaches to the Management of Tension and Stage Fright in Music Performance. Journal of Research in Music Education, 35(3), 143-153.

Lucena, R. (2008). El profesor que programa: la programación como instrumento de trabajo. En J. C. Sánchez Huete (Ed.), Compendio de Didáctica General. Madrid: Editorial CCS.

Magnusson, M. S. (2005). Understanding social interaction: discovering hidden structure with model and algorithms. En L. Anolli, S. Duncan Jr., M. S. Magnusson \& G. Riva (Eds.), ítepiThe hidden structure of interaction: from neurons to culture patterns.

Marín, C., Pérez, P., \& Hallam, S. (2009). The use of musical scores in order to perform: An exploratory study with flute players. En A. Williamon, S. Pretty \& R. Buck (Eds.), Proceedings of the International Symposium on Performance Science 2009. Utrecht, The Netherlands: European Association of Conservatoires (AEC).

Parkes, K. A. (2010). Recent research in applied studio instruction: Practice time and strategies. Musical perspectives, 1, 5-18.

Pitts, S. E., Davidson, J. W., \& McPherson, G. E. (2002). Developing effective practice strategies: Case studies of three young instrumentalists. En G. Spruce (Ed.), Aspects of teaching secondary music: perspectives on practice. London: RoutledgeFalmer.

Quera, V. (1993). Muestreo y registro observacional. En M.T. Anguera (Ed.), J. Behar, A. Blanco, M.V. Carreras, J.L. Losada, V. Quera y C. Riba, Metodología observacional en la investigación psicológica. Vol. 1. (2th ed.). Barcelona: Promociones y Publicaciones Universitarias.

Quera, V., Bakeman, R., \& Gnisci, A. (2007). Observer agreement for event sequences: Methods and software for sequence alignment and reliability estimates. Behavior Research Methods, 39(1), 39-49.

Renwick, J. M., \& McPherson, G.E. (2002). Interest and choice: Student-selected repertoire and its effect on practicing behavior. British Journal of Music Education, 19(2), 173-188.

Sloboda, J. A., Davidson, J. W., Howe, M. J. A., \& Moore, D. G. (1996). The role of practice in the development of performing musicians. British Journal of Psychology, 87, 287-309.

Tójar, J. C. (1993). Concordancia en los registros de observación. Calidad de la investigación educativa en metodología observacional. Barcelona: Promociones y publicaciones universitarias. 
Tripiana, S. (2012). Estrategias eficaces de práctica instrumental. Música y Educación: Revista internacional de pedagogía musical, 91, 64-72.

Tripiana, S. (2016). Estrategias de motivación durante el aprendizaje musical. Revista internacional de educación musical, 4, 25-33. DOI: 10.12967

Weick, K. E. (1968). Systematic observational methods. The handbook of social psychology, 2, $357-451$.

Manuscrito recibido el 22/12/2016 y evaluado anónimamente. Aceptado para su publicación el 13/06/2016. 